\title{
Towards European harmonisation of healthcare for patients with rare immune disorders: outcome from the ERN RITA registries survey
}

\author{
Riccardo Papa ${ }^{1}$, Andrew Cant ${ }^{2}$, Christoph Klein ${ }^{3}$, Mark A. Little ${ }^{4}$, Nico M. Wulffraat ${ }^{5}$, Marco Gattorno ${ }^{6}$, \\ Nicolino Ruperto ${ }^{7 *}$ and on behalf of ERN RITA Council
}

\begin{abstract}
The Rare Immunodeficiency, Autolnflammatory and Autolmmune Disease (RITA) network is a European Research Network (ERN) that brings together the leading centres for rare immune disorders. On April 2018 an online survey was sent to all RITA members in order to facilitate the harmonization of data collection in rare immune disorders registries. Currently, as many as 52 different registries collect data on rare immune disorders, of whom 30 (58\%) are dedicated primarily to autoimmune diseases, 15 (29\%) to primary immunodeficiencies and 12 (23\%) to autoinflammatory disorders. Improving data on patient safety, outcome, and quality of life measures is warranted to unfold the full potential of RITA registries.
\end{abstract}

Keywords: Primary immunodeficiency, Autoinflammatory diseases, Autoimmune disorders, European research network, Disease registry

\section{Background}

Immunology is a young discipline, originating from the discovery of innate and adaptive immune responses in the last century [1]. The field has evolved rapidly from the identification of primary immunodeficiencies (PID) predisposing to infection, to the recognition of specific rare immune disorders with disturbed immunoregulatory balances, called Autoinflammatory disorders (AID), and others with defective tolerance to self-antigens, also known as Autoimmune diseases (AI) [2-4]. Thus, errors in the immune system can result not only in specific clinical entities, but also in conditions with two or three features of these pathologic processes $[5,6]$.

A multidisciplinary care for patients with rare immune disorders (RID) using complex diagnostic

* Correspondence: nicolaruperto@gaslini.org; https://www.printo.it 7 UOSID Centro Trial, PRINTO, IRCCS Istituto Giannina Gaslini, Genoa, Italy Full list of author information is available at the end of the article evaluation and highly specialized therapies is nowadays required. For example, genome-wide analysis platforms and functional immune assays are rapidly developing diagnostic tests shared across all subthemes and usually not available in all centres [7, 8]. Polyvalent immunoglobulin therapy has revolutionised the outlook for antibody deficient patients, specific anti-cytokine (anti-TNF, anti-IL-1 etc) treatments have transformed the lives of patients with AID and $\mathrm{AI}$, and stem cell and gene-based therapies, originally for PID, are now being applied for all the RID and for the first time enable patients to be completely cured with no need for ongoing medical care [9-11].

\section{RITA proposal}

RITA is a European research network (ERN) that brings together the leading European centres with expertise in diagnosis and treatment of RID. ERNs are 
virtual networks involving healthcare providers (HCP) and family organization across Europe to facilitate discussion on complex or rare diseases that require highly specialised treatment, concentrating knowledge and resources (https://ec.europa.eu/health/ern_en) [12]. The foundation of RITA has been developed from the resources of successful, already existing, highly specialised international scientific societies, registries and websites, and in particular European Society for Immune Deficiencies (ESID) with ESID registry, Paediatric Rheumatology European Society (PRES), International Society for Systemic AutoInflammatory Diseases (ISSAID), Paediatric Rheumatology International Trials Organisation (PRINTO) with EUROFEVER and PHARMACHILD projects, European Vasculitis Society (EUVAS), and BEHCET International.

The main aims of RITA are: i) to provide state of the art for comprehensive clinical care for children and adults with RID, harmonizing diagnostic and therapeutic guidelines across Europe, thus ensuring for every patient an equal access to excellent expertise and care and reducing patient search of crossborder healthcare; ii) to establish sustainable alliances within European centres to accelerate diagnosis, improve access to treatment, and develop transitional care for patients with RID, maximizing the costeffective use of resources and facilitating mobility of expertise; iii) to ensure the correct codification of quality control on diagnostic tests and targeted therapy, establishing a common tool for pharmacovigilance in these rare conditions; iv) to reinforce epidemiological surveillance and basic research on RID; v) to train future leaders in the field, securing their sustainability in an international perspective, and promote the awareness of RID between clinicians, carers, patients, family organizations and politicians, advocating better resources and measure to ensure early diagnosis by screening and enhanced symptom recognition.

Thus, providing a common shared medical platform for all affected patients and HCPs, independently of nationality and ethnicity, is a major attempt of the network $[13,14]$. We report herein the results of an international survey within RITA members about all existing registries and research networks with the aim to harmonise and facilitate future data merging for research and clinical purposes.

\section{Methods}

Each RITA member was invited with a formal email to participate in the present survey (Additional file 1). In brief, each member was asked about personal involvement in any registry projects and covered role (coordinator, participant or just knower). We collected data about registries name, website, boundaries extension (national or international with involved countries), considered group of diseases (PID, AID, and/or AI), number of enrolled patients, and types of collected data. Members shared registry protocol and/ or case report form when possible.

All data were collected and analysed using the Excel program. Descriptive data were reported as absolute numbers and percentages for categorical variables.

\section{Survey results}

The online survey was sent to all 126 RITA members during April 2018, of whom 45 are HCPs and 8 are patients and family organizations (Table 1 ). Informative responders were 75 (60\%) because 15 members delegated the response to other members of the network. Collectively, 27 members are coordinating 25 registries; 53 members are participating in 38 registries, and 27 members knew the existence of 16 registries without participating. Only two members are not involved in any registries.

Data about 52 different registries were collected across 14 European countries (Table 2). Almost 50\% of registries collect data on AI, while others are dedicated to PID or AID (respectively 15 registries, 29\%, and 12 registries, 23\%). Fifteen registries (29\%) enrolled patients with a single specific disorder, in particular three registries for monogenic forms of systemic lupus erythematosus, two registries for Kawasaki disease or Behcet disease, and single registry for juvenile dermatomyositis, juvenile systemic sclerosis, juvenile idiopathic arthritis (JIA)-related uveitis, systemic JIA, Blau syndrome, sarcoidosis, GuillainBarre syndrome, and myasthenia gravis.

More than 55,000 patients with RID are enrolled in a disease registry in Europe (Table 2). The majority of registries $(36 ; 69 \%)$ enrols patients from national boundaries and only one registry collect data from two countries (UKIVAS registry). Among the international registries, five collect data on PID (ESID, EBMT, SCETIDE, PCID and HLH registry), four with AI (Pharmachild, BrainWorks, EULAR web library, and JIR cohort), and three are devoted to AID (Eurofever, Infevers, and ImmunAID). The ESID registry and JIR cohort also collect data on AID. Other international registries are devoted to a single specific disorder, i.e. monogenic forms of systemic lupus erythematosus, juvenile systemic sclerosis, Behcet disease and Blau syndrome.

Data usually collected in these registries are demography, diagnosis, clinical manifestations, laboratory tests and treatment, while genetic and imaging data are less frequently reported (respectively in 39 and 
Table 1 Registries survey results overview

\begin{tabular}{|c|c|}
\hline $\begin{array}{l}\text { Are you aware of any Registry of Diseases covered by the ERN } \\
\text { RITA? }\end{array}$ & $\begin{array}{l}\text { Number } \\
(\%)\end{array}$ \\
\hline Responder RITA members & $\begin{array}{l}90 / 126 \\
(71)\end{array}$ \\
\hline Responder HCPs & $39 / 45(87)$ \\
\hline Responder family organizations & $4 / 8(50)$ \\
\hline Delegate to other RITA members & 15/90 (17) \\
\hline Countries & 14 \\
\hline Informative responders & $\begin{array}{l}75 / 126 \\
(60)\end{array}$ \\
\hline Coordinators & $27 / 75(36)$ \\
\hline Participants & $53 / 75(71)$ \\
\hline Knew registry without participating & $27 / 75(36)$ \\
\hline Not knew any registry & 2/75 (3) \\
\hline Registries & 52 \\
\hline \multicolumn{2}{|l|}{ Is it a National or International Registry? } \\
\hline International & $16 / 52(31)$ \\
\hline National & $36 / 52(69)$ \\
\hline \multicolumn{2}{|l|}{ Which area of diseases is covered by the Registry you are involved? } \\
\hline Autoimmunity & $29 / 52(56)$ \\
\hline Primary immunodeficiency & 15/52 (29) \\
\hline Autoinflammation & 20/52 (39) \\
\hline Only autoinflammation & $12 / 52(23)$ \\
\hline \multicolumn{2}{|l|}{ What type of data are you collecting? } \\
\hline Demography & $42 / 52(81)$ \\
\hline Diagnosis & $45 / 52(87)$ \\
\hline Signs and symptoms & $40 / 52(77)$ \\
\hline Therapy & $39 / 52(75)$ \\
\hline Safety & $28 / 52(54)$ \\
\hline Genetic & 20/52 (39) \\
\hline Laboratory & $35 / 52(67)$ \\
\hline Imaging & $5 / 52(10)$ \\
\hline Biobank & $15 / 52(29)$ \\
\hline
\end{tabular}

$10 \%$ of registries). A treatment safety profile is reported in 29 registries (56\%). Collectively, fifteen biobank are counted.

\section{Discussion}

RITA intends to ensure that all patients with RID are included in Europe wide registries to facilitate harmonization of patient care, audit of compliance with agreed guidelines and to create a platform for research.

The present survey of registries clearly shows how the network needs a common plan for inventory the clinical data about patients with RID. Furthermore, key parameters of patient safety, including the use of medicines and medical technologies, communication issues and breaches in continuity of care, as well as outcome data (e.i. mortality, morbidity and disease complications) or quality of life measures are not usually reported in the current registries. Differences in the number of patients registered across countries may be related to a different capacity of each HCP to collect and enter the data in the exiting registries. RITA aims at supporting these HCPs, promoting registry-oriented researches and looking for funding to supports to registry users.

In this line, the European Commission Department for Health \& Food Safety recently developed the European Rare Disease Registry Infrastructure (ERDRI) as part of the European Platform on Rare Diseases Registration (EU RD Platform, available at https://eu-rd-platform.jrc.ec.europa.eu). By promoting interoperability between data sources, the EU RD Platform helps reaching the necessary critical numbers to conduct studies and research on rare disease. The major objective of the project is to tackle the enormous fragmentation of patient data contained in hundreds of patient registries across Europe. The ERDRI strategy is to create an inventory of registries about rare diseases, in which 27 characteristics for each registry were considered, and a list of 16 minimum data element to be registered by all registries across Europe, providing instructions on how and in which format each data element should be registered. A phonetic hashing to prevent duplicate registration of patients has been proposed and freely available at https://eupid.eu/.

The next RITA aim will be to support the conversion of all existing registries about RID to the ERDRI format to ensure their interoperability with other European rare disease registries with the belief that cross fertilisation of ideas would enable these registries to be further developed than would be otherwise. In fact, disease registries play an important role in the development of orphan drugs, so essential to the treatment of patients with rare disorders. RITA will seek to facilitate collaboration with industry for the further development of such treatments. It is expected that other registries in the meanwhile identified (e.g. the Spanish Registry of Primary Immunodeficiencies REDIP), will be identified and invited to participate to any future initiative.

\section{Conclusions}

The survey highlighted the pivotal role of national and international organizations in Europe to collect and organize clinical data on immune diseases, allowing the rapidly growing knowledge on these rare disorders, creating research networks and providing significant numbers of data to support new discoveries in the field. RITA network could improve the 


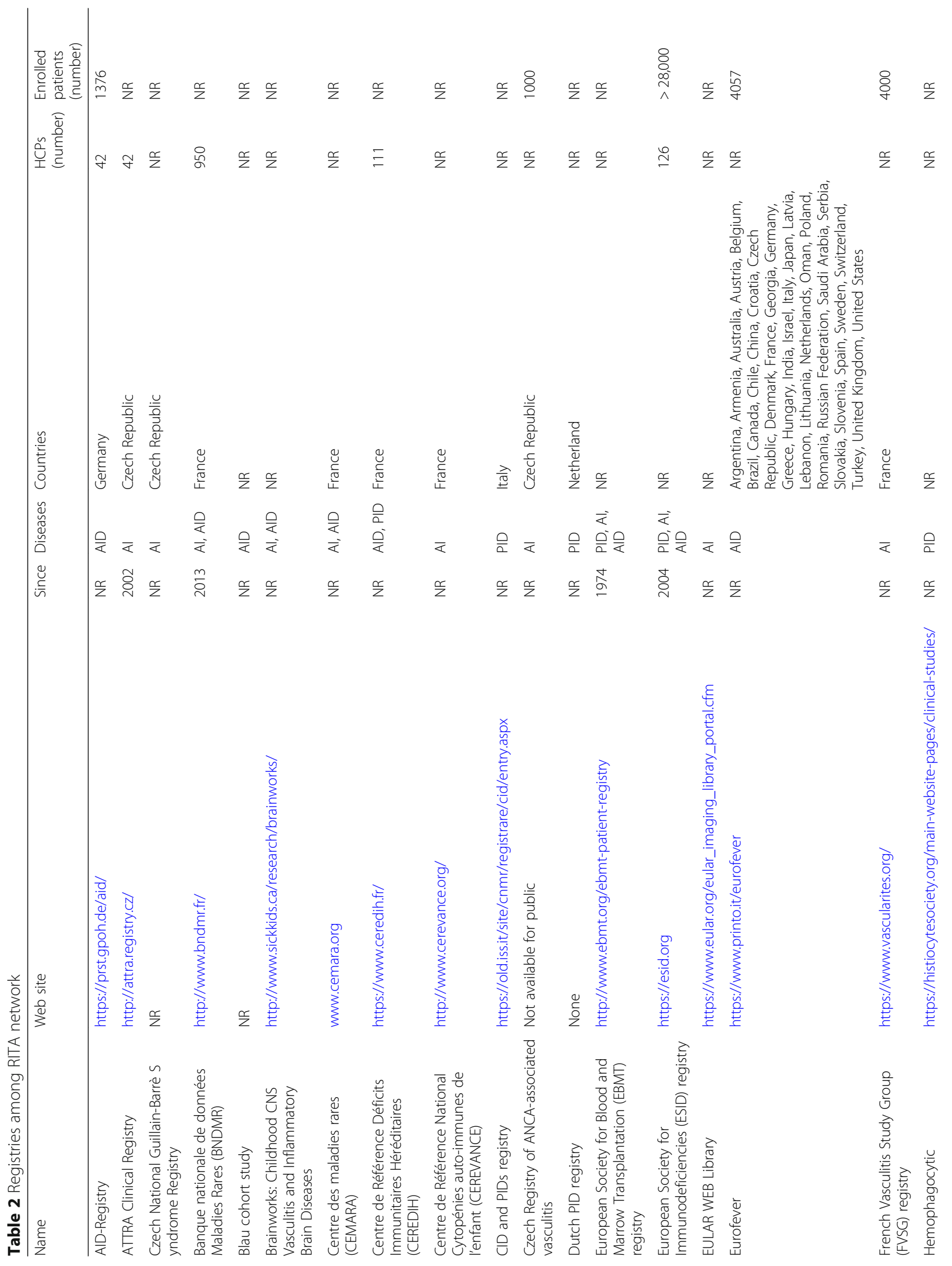




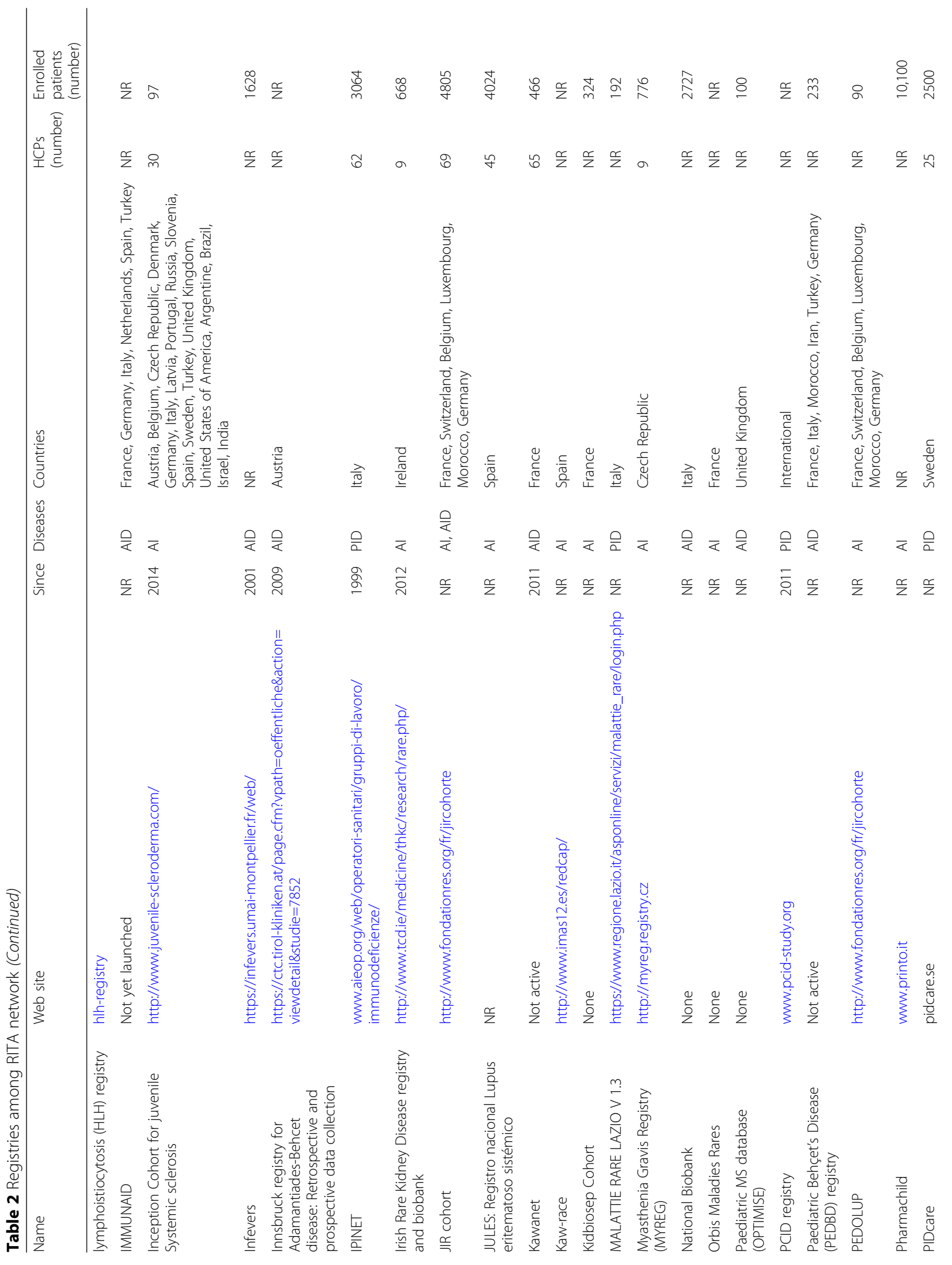




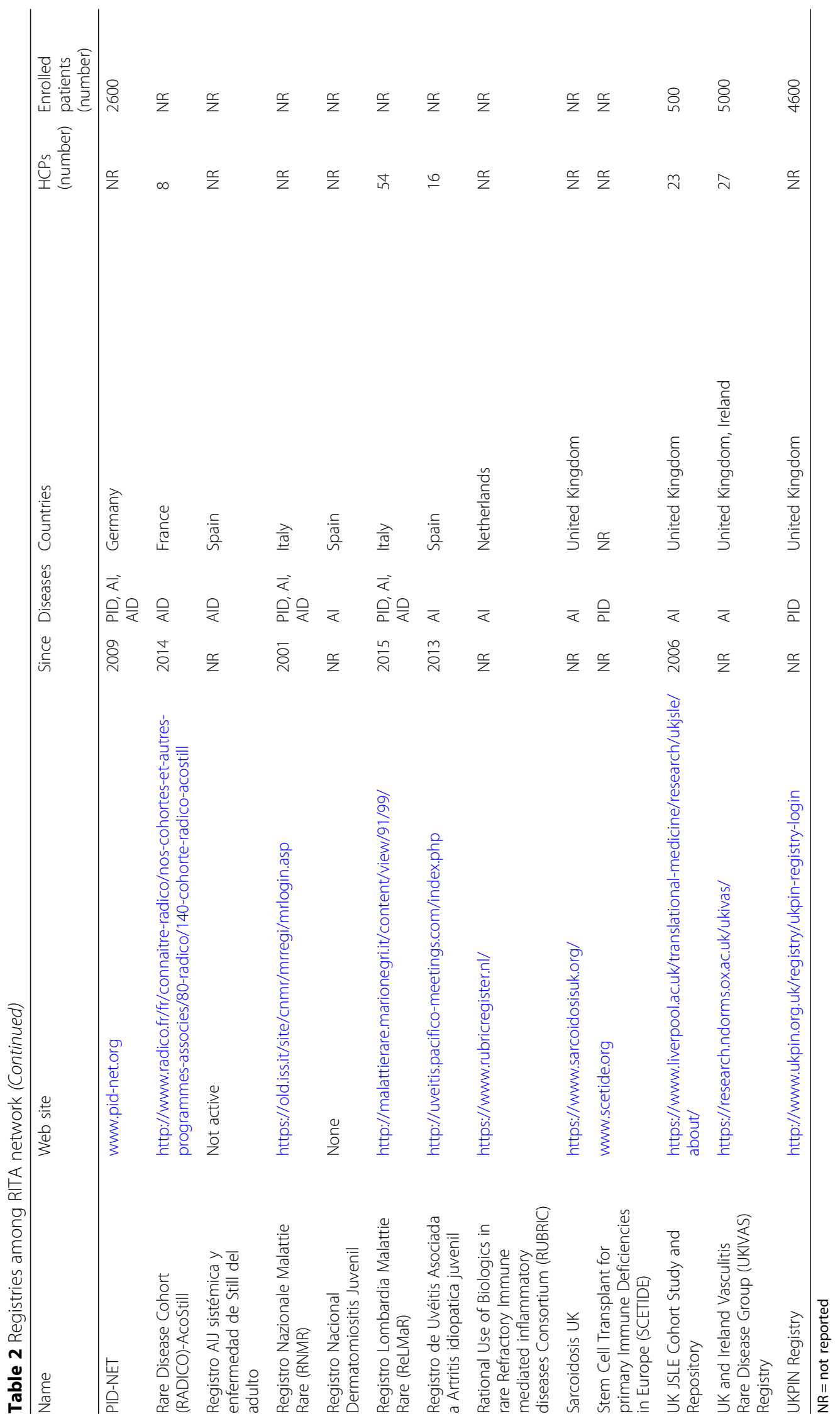


coordination of these numerous entities, supporting initiatives of collaboration and cooperation. As a first attempt, the present survey revealed that the collection of key parameters about patient safety, as well as outcome data and quality of life measures should be improved among the registries of RITA network.

\section{Supplementary information}

Supplementary information accompanies this paper at https://doi.org/10. 1186/s13023-020-1308-x.

Additional file $\mathbf{1}$. The registries survey sheet.

\begin{abstract}
Abbreviations
Al: Autoimmune diseases; AID: Autoinflammatory disorders; ERDRI: European Rare Disease Registry Infrastructure; ERN: European Reseach Network; ESID: European Society for Immune Deficiencies; EU RD Platform: European Platform on Rare Diseases Registration; EUVAS: European Vasculitis Society; HCP: Health Care Provider; ISSAID: International Society for Systemic AutoInflammatory Diseases; JIA: Juvenile Idiopathic Arthritis; PID: Primary immunodeficiencies; PRES: Paediatric Rheumatology European Society; PRINTO: Paediatric Rheumatology International Trials Organisation; REDIP: Spanish Registry of Primary Immunodeficiencies; RID: Rare immune disorders
\end{abstract}

\section{Acknowledgements}

The authors would like to thanks Eugenia Mosci, MPol and Elisa Patrone, MA research assistants of the Paediatric Rheumatology International Trials Organisation (PRINTO, www.printo.it) for the management of the survey. This survey has been supported by the European Reference Network for Rare Immunodeficiency, Autoinflammatory and Autoimmune Diseases, which is partly co-funded by the European Union with in the framework of the Third Health Programme "ERN-2016-Framework Partnership Agreement 20172021".

\section{Authors' contributions}

RP performed the analysis and interpretation of data and drafted the manuscript; MG and NR concepted and designed the manuscript, revised it critically, and given final approval of the version to be published. All authors read and approved the final manuscript.

\section{Authors' information}

NR and RP drafted the first and subsequent version of the manuscript. All authors contributed to the planning of the survey. All authors revised critically and approved the content of the final manuscript.

\section{Funding}

This study was supported by the ERN Specific Grant Agreements Year 2 (ERN-SGA-2017).

\section{Availability of data and materials}

The datasets used and/or analysed during the current study are available from the corresponding author on reasonable request.

\section{Ethics approval and consent to participate}

Experts involved were invited to complete the survey by clicking on a hyperlink in the email that directed them to the web-based survey. The results discussed in this manuscript are based on a voluntary survey and did not involve any experimental research on human subjects. Hence, ethical approval and informed consent procedures were not applicable.

\section{Consent for publication}

The article is original, has not already been published in a journal, and is not currently under consideration by another journal. The authors agree to the terms of the BioMed Central Copyright and License Agreement and Open Data policy.
All authors of the manuscript have read and agreed to its content and are accountable for all aspects of the accuracy and integrity of the manuscript in accordance with ICMJE criteria.

\section{Competing interests}

The authors declare that they have no competing interests.

\section{Author details}

${ }^{1}$ Clinica Pediatrica e Reumatologia, IRCCS Istituto Giannina Gaslini, Genoa, Italy. ${ }^{2}$ Great North Children's Hospital \& Institute for Cellular Medicine, University of Newcastle, Newcastle upon Tyne, UK. ${ }^{3}$ Department of Pediatrics, Dr. von Hauner Children's Hospital, University Hospital, LMU Munich, Munich, Germany. ${ }^{4}$ Trinity Health Kidney Centre, Tallaght University Hospital, Dublin, Ireland. ${ }^{5}$ Department of Pediatrics, Section Pediatric Rheumatology, Wilhelmina Children's Hospital, University Medical Centrum Utrecht, Utrecht, Netherlands. ${ }^{6}$ Centre for Autoinflammatory Diseases and Immunodeficiencies, IRCCS Istituto Giannina Gaslini, Genoa, Italy. 7 UOSID Centro Trial, PRINTO, IRCCS Istituto Giannina Gaslini, Genoa, Italy.

Received: 9 April 2019 Accepted: 14 January 2020

Published online: 30 January 2020

\section{References}

1. Wong M. What has happened in the last 50 years in immunology. J Paediatr Child Health. 2015;51(2):135-9.

2. Picard C, Al-Herz W, Bousfiha A, Casanova JL, et al. Primary immunodeficiency diseases: an update on the classification from the International Union of Immunological Societies Expert Committee for primary immunodeficiency 2015. J Clin Immunol. 2015;35(8):696-726.

3. Manthiram K, Zhou Q, Aksentijevich I, Kastner DL. The monogenic autoinflammatory diseases define new pathways in human innate immunity and inflammation. Nat Immunol. 2017;18(8):832-42.

4. Davidson A, Diamond B. Autoimmune diseases. N Engl J Med. 2001;345(5): 340-50.

5. Fischer A, Provot J, Jais JP. Autoimmune and inflammatory manifestations occur frequently in patients with primary immunodeficiencies. J Allergy Clin Immunol. 2017;140(5):1388-93.

6. Damgaard RB, Walker JA, Marco-Casanova P, et al. The Deubiquitinase OTULIN is an essential negative regulator of inflammation and autoimmunity. Cell. 2016;166:1215-30.

7. Hardwick SA, Deveson IW, Mercer TR. Reference standards for nextgeneration sequencing. Nat Rev Genet. 2017;18(8):473-84.

8. Castro C, Gourley M. Diagnostic testing and interpretation of tests for autoimmunity. J Allergy Clin Immunol. 2010;125:S238-47.

9. Wolfe RM, Ang DC. Biologic therapies for autoimmune and connective tissue diseases. Immunol Allergy Clin N Am. 2017:37(2):283-99.

10. Ewart DT, Peterson EJ, Steer CJ. Gene editing for inflammatory disorders. Ann Rheum Dis. 2019:78(1):6-15.

11. Freeman AF. Hematopoietic stem cell transplantation in primary Immunodeficiencies beyond severe combined immunodeficiency. J Pediatric Infect Dis Soc. 2018;7:S79-82.

12. Heon-Klin V. European reference networks for rare diseases: what is the conceptual framework? Orphanet J Rare Dis. 2017;12(1):137.

13. Beukelman T, Anink J, Berntson L, et al. A survey of national and multinational registries and cohort studies in juvenile idiopathic arthritis: challenges and opportunities. Pediatric Reumatology. 2017;15:31.

14. Dolezalova P, Anton J, Avcin T, et al. The European network for care of children with paediatric rheumatic diseases: care across borders. Rheumatology (Oxford). 2019;21:1188-95.

\section{Publisher's Note}

Springer Nature remains neutral with regard to jurisdictional claims in published maps and institutional affiliations. 